\title{
REMARKS CONCERNING GRAPHICAL MODELS FOR TIME SERIES AND POINT PROCESSES*
}

\author{
David R. Brillinger**
}

\section{Resumo}

Uma rede estatística é uma coleção de nós representando variáveis aleatórias e um conjunto de arestas que ligam os nós. Um modelo estocástico por isso é chamado um modelo gráfico. Estes modelos, de gráficos e redes, são particularmente úteis para examinar as dependências estatísticas baseadas em condiçōes do tipo das que ocorrem frequentemente em economia e estatística. Neste artigo as variáveis aleatórias dos nós serão séries temporais ou processos pontuais. Os casos de gráficos direcionados e não-direcionados são apresentados.

\section{Abstract}

A statistical network is a collection of nodes representing random variables and a set of edges that connect the nodes. A probabilistic model for such is called a graphical model. These models, graphs and networks are particularly useful for examining statistical dependencies based on conditioning as often occurs in economics and statistics. In this paper the nodal random variables will be time series or point processes. The cases of undirected and directed graphs are focussed on.

Palavras-Chave: Graphical model, network, point process, time series

Códig• JEL: C32, C35, C34

* Based on a talk presented at the Sixth School of Time Series and Econometrics, Vitória, ES - 31 July to 2 August, 1995. Prepared with the partial support of the National Science Foundation Grant DMS-9300002 and Office of Naval Research Grant ONR-N00014-9-1.

** Department of Statistics, University of California, Berkeley, CA - 94720, USA.

I gratefully thank Silvia Lopes Troya for her help in translating this Alstract to Portuguese.

\begin{tabular}{llll}
\hline R. de Econometria & Rio de Janeiro & v. $16, \mathrm{n}^{\mathrm{O}} 1$, pp. $1-23$ & Abril 1996 \\
\hline
\end{tabular}


Graphical models for time series

\section{Introduction.}

Graphical models, that is probability models on networks of vertices and edges, are experiencing a surge of research development today. One can point to the books by Whittaker (1990) and by Edwards (1995) as well as to many papers, for example those listed in the References section. The study of graphical models has become important in the field of statistics and in the social sciences generally. It can be anticipated that such models will prove useful in addressing basic questions arising in economics concerning structural and causal modelling.

Given its historical roots in path analysis, simultaneous equations, structural and finally recursive models, research on graphical models is of importance to economists and econometricians. An economy can be viewed as a vast system or network of interconnected processes. In various circumstances the connections (or edges) are of particular interest and the processes are either time series or point processes. Structural approaches, e.g. via time series or econometric models, are well known to economists and econometricians, see e.g. Granger (1980), Hendry and Richard (1983) and Harvey (1989). This paper focuses on some of what graphical models have to offer.

As an example of a conceptual economic network, of some historical interest, one can mention the Philips machine, Philips (1950), Swade (1995). Pictures of the machine may be found in those references. Figure 1 presents a graph of Philips' Simple Model. The machine was meant to represent the behaviour of the British economy in the late forties. The arrows indicate the direction of the flow of liquid in the machine. Water is pumped to the top and flows downwards. Savings (S) are siphoned off into a separate loop and fall into a tank ( $\left.\mathrm{M}^{\prime}\right)$. Portions of these rejoin the main flow as expenditure (E) and investment (I). The net flow at the bottom accumulates in a tank. The level of the tank represents the working balance (M) required for a given level of economic activity. The liquid is pumped back to the top. The flow of water represents the movement of money 
and the accumulation of money is represented by the water collecting in tanks. Results are read off from scales located at various places. Flow is initiated, for example, by altering expenditure. The series involved here are (continuous) time series.

Less often economic data of point process form, $\left\{\tau_{j}\right\}$, occur. That is the measurements are the times of occurence of certain events, such as the times of change of a country's prime interest rate or the ticker of stock sales. Economic data may also be of marked point process form, consisting of times and associated values (marks), e.g. the times of change of the prime rate together with the value of the new rate, $\left\{\left(\tau_{j}, M_{j}\right)\right\}$. In this paper time series and point processes are labelled by the nodes of a graph and corresponding statistical models discussed. The models may involve the processes at vertices $u$ and $\nu$ being conditionally independent given the remaining vertices. Such a question may be formulated as involving the existence or nonexistence of an edge connecting vertices $u$ and $\nu$. Recognizing such structure allows decomposition of the estimation problem into simpler components, in various cases. The question of measuring the strengths of connections present is also addressed in this article.

The use of graphs to represent statistical models dates back at least to Wright (1921) and his work with path models. One can also mention the graphs of Tinbergen (1939), page 138, for causal connections between disturbances and profits and of Wold (1956), page 45 , for a demand-supply model. Another example is provided by the schematic diagrams so often employed in systems analysis. Networks are present in compartment analyses (see Jacquez (1972)). Here substances flow between and are retained in certain compartments. Influence diagrams and Bayesian networks are related topics, see Smith (1989), Normand and Tritchler (1992). Pearl (1995) presents a framework for causality and the manipulation of probabilities associated with such graphical models. Causal analysis is further discussed in Glymour et al. (1987). An example of a marked point process is provided by a queuing network. Here there is a 
set of interconnected nodes, each node consisting of a queue, where customers wait for service, and there are one or more servers. (See Brémaud (1981), for example.)

This present work constitutes a simple begining discussion of the time series and point process cases of graphical models. It is to be noted that the meanings of graphical representations differ between authors and applications, and this needs to be kept in mind when reading the literature.

The layout of the paper is: first some basic concepts of graph theory and graphical models are presented, then consideration turns to the case of continuous and discrete ordinary random variables, finally the time series and point process cases are addressed. The paper ends with a discussion and summary.

\section{Graphical Models.}

A graphical model is a statistical model embodying a set of conditional independence relationships which may be summarized by means of a graph. This section presents some general concepts and then moves on to the specific cases of undirected and directed graphs. The goal, for inferential purposes, is the likelihood function. General references include: Whittaker (1990), Lauritzen et al. (1990), Wermuth and Lauritzen (1990), Cox and Wermuth (1993), Edwards (1995).

A graph is a pair $G=(V, E)$ where $V$ is a finite set of nodes (or vertices) and $E$, the set of edges, is a subset of $V \times V$, the ordered pairs of distinct nodes. Figure 2 provides an example with 4 nodes and 2 edges. An edge $(\alpha, \beta)$ in $E$ is called undirected if both $(\alpha, \beta)$ and $(\beta, \alpha)$ are in $E$. An undirected edge is indicated by a line. A graph is simple if there are no multiple edges or loops. (This will be assumed throughout the paper). This is the case in Figure 2. A clique is a maximal set of nodes, joined to each other. For Figure 2 the cliques are: $\{1\},\{2,4\},\{3,4\}$. An undirected graph is one with only undirected edges. An edge $(\alpha, \beta)$ is called directed if $(\alpha, \beta)$ is 
in $E$, but not $(\beta, \alpha)$. A directed edge is denoted by an arrow $\rightarrow$. A directed graph is one in which all edges are directed. Figure 3 and 4 provides examples of directed graphs. There are two edges in Figure 3 and three in Figure 4. An acyclic directed graph has no paths beginning and ending at the same node. The graphs of Figure 3 and 4 are acyclic, while the graph of Figure 1 is cyclic (feedback is present).

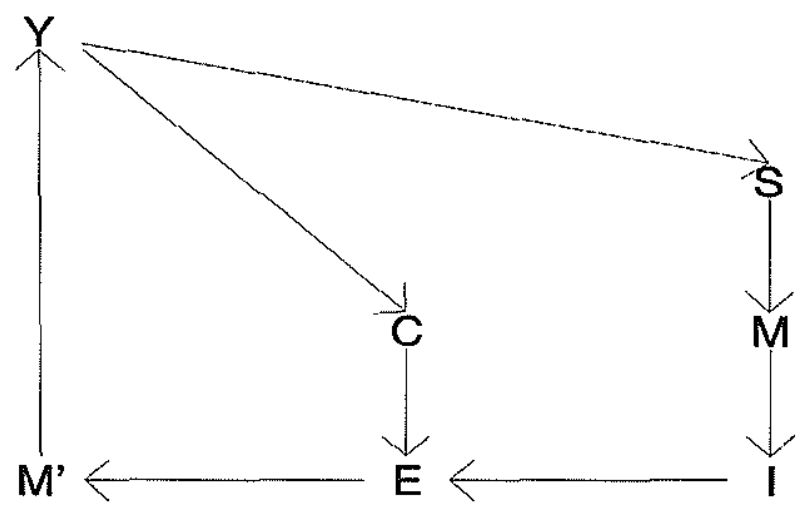

Figure 1. Philips Simple Model. C: consumption, E: total expenditure, I: investment expenditure, $\mathrm{M}$ : surplus balances, $\mathrm{M}^{\prime}$ : transfer balances, S: savings, Y: income.

1

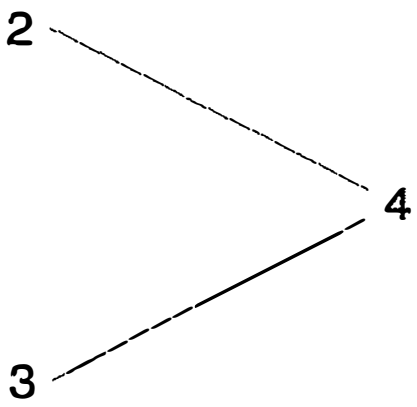

Figure 2. Example of an undirected graph, with 4 nodes. 


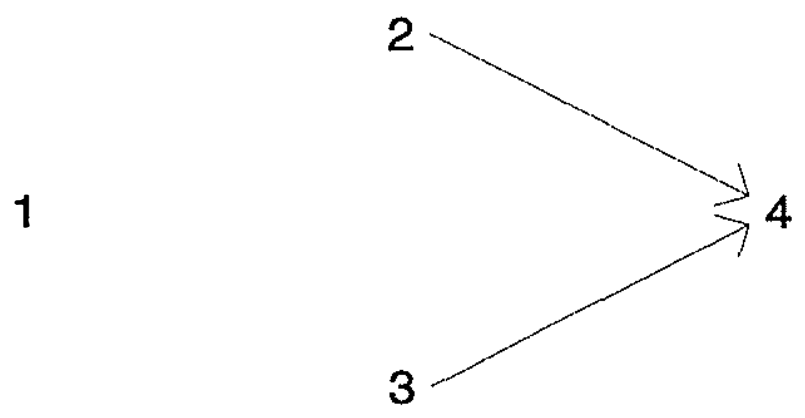

Figure 3. Example of a directed graph, with 4 nodes and 2 directed edges.

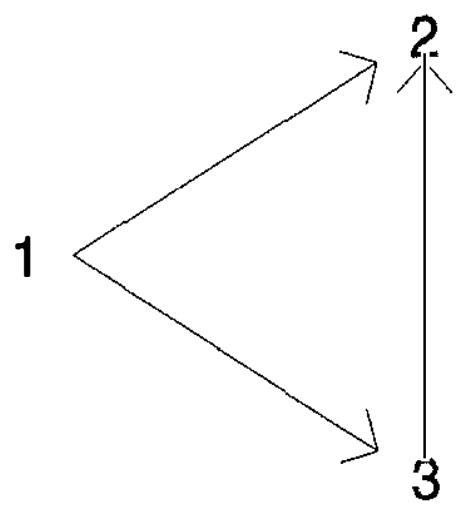

Figure 4. Example of a directed graph, with 3 nodes and 3 directed edges.

\section{1.' Undirected Graphs.}

A set $B$ of vertices separates the sets $A$ and $C$ if all paths from vertices in $A$ to $C$ pass through $B$. An undirected graph possesses the global Markov property if for any triple $(A, B, C)$ of disjoint subsets 
of $V$, such that $B$ separates $A$ from $C$, the variate $\left(Y_{a}\right)_{a \in A}$ is statistically independent of the variate $\left(Y_{c}\right)_{c \in C}$ given the variate $\left(Y_{b}\right)_{b \in B}$. Conditions for this occuring may be found in Lauritzen at al. (1990). In broad circumstances it may be shown that the probability density of $\mathrm{Y}$ then has the form

$$
f(\mathbf{y})=\prod_{\gamma} \psi_{\gamma}(\mathrm{y})
$$

where the product is over all the cliques $\gamma$ of $G$. For the graph of Figure 2 this means

$$
f\left(y_{1}, y_{2}, y_{3}, y_{4}\right)=g\left(y_{1}\right) h\left(y_{2}, y_{4}\right) i\left(y_{3}, y_{4}\right)
$$

Here the variates $Y_{2}$ and $Y_{3}$ are independent given the variate $Y_{4}$. This type of conditional independence is typical of undirected graphs. Taking $f(\cdot)$ to be say a multivariate normal density, the factorization can indicate directly parameter values which need to be set to 0 .

For the case of undirected graphs, Geiger and Pearl (1993) show that every axiom for conditional independence is an axiom for graph separation and that every graph represents a consistent set of independence and dependence constraints. They conclude that graphs provide a safe language for encoding statistical dependencies.

There is also a form of the results for discrete variates, with the densities replaced by probability mass functions.

\subsection{Directed Graphs.}

Consideration next focuses on directed graphs in the acyclic case. In empirical analyses the arrow may represent a direction of influence or of causation or of a variate value at an earlier time point. For $a$ and $b$ vertices, $a$ is a parent of $b$ if there is a directed edge $a \rightarrow b$. The set of parents of the vertex $b$ is denoted $p a(b)$. 
A directed acyclic graph possesses the directed global Markov property if the density admits a recursive factorization,

$$
f(\mathbf{y})=\prod_{\nu \in V} k_{\nu}\left(\mathrm{y}_{\nu}, \mathrm{y}_{p a(\nu)}\right)
$$

for some functions $k_{\nu}$. The term $k_{\nu}\left(y_{\nu}, y_{p a(\nu)}\right)$ has the interpretation of the conditional density of $Y_{\nu}$ given $\mathrm{Y}_{p a(\nu)}$. Conditions for this property are given in Lauritzen et al. (1990).

For the graph of Figure 3 the factorization is:

$$
f_{1}\left(y_{1}\right) f_{2}\left(y_{2}\right) f_{3}\left(y_{3}\right) f_{4}\left(y_{4} \mid y_{2}, y_{3}\right)
$$

with $p a(4)=\{2,3\}$. For Figure 4 it is

$$
f_{1}\left(y_{1}\right) f_{2}\left(y_{2} \mid y_{1}, y_{3}\right) f_{3}\left(y_{3} \mid y_{1}\right)
$$

with $p a(2)=\{1,3\}$ and $p a(3)=\{1\}$. One might consider the hypothesis

$$
f_{2}\left(y_{2} \mid y_{1}, y_{3}\right)=f_{2}\left(y_{2} \mid y_{1}\right)
$$

i.e. that there is no direction connection of vertex 3 to vertex 2 . The expressions above lead directly to likelihood functions useful for inferential purposes.

\subsection{Strength of Connections.}

Suppose there is a graphical model. One may wish to describe the strength of connection associated with a particular edge. Two (related) measures may be considered. The first is the mutual information, the second is the change in deviance when an edge is removed from the model. The measures will be seen to be quite related.

Consider continuous random variables $X$ and $Y$ with joint density $p(x, y)$ and marginals $p_{X}(x)$ and $p_{Y}(y)$. The mutual information between $X$ and $Y$ is

$$
I_{X Y}=\iint p(x, y) \log \frac{p(x, y)}{p_{X}(x) p_{Y}(y)} d x d y .
$$


It is seen to be 0 in the case that $X$ and $Y$ are independent and $>0$ otherwise. For some discussion of this quantity see Granger and Hatanaka (1964), Kanter (1979) and Parzen (1983).

The mutual information may be related to the differential entropy of a random variable. For a random variable $Y$, with density $p_{Y}(y)$, the differential entropy is

$$
H_{Y}=-\int p_{Y}(y) \log p_{Y}(y) d y
$$

and one sees that

$$
I_{X Y}=H_{Y}-H_{Y \mid X}
$$

in particular.

The deviance of a stochastic model is minus twice the loglikelihood, $-2 \log p_{Y}(y)$. The change in deviance that results from fitting a submodel proves a useful quantity. Consider fitting the submodel of independence, $p(x, y)=p_{X}(x) p_{Y}(x)$, then the change in expected deviance is

$$
\begin{aligned}
& -2 \iint p_{X}(x) p_{Y}(y) \log p_{X}(x) p_{Y}(y) d x d y \\
& +2 \iint p(x, y) \log p(x, y) d x d y \\
& =2 I_{X Y} .
\end{aligned}
$$

Deviance change and mutual information are thus closely related and either may be considered as a measure of strength of a connection.

In the case of a graphical model one can study the change in deviance when one fits, both including a particular edge and not. The values may be added to a graph in a presentation of the results of the analysis. The edge might be directed or not. Some computer programs are available for carrying out the computations, e.g. MIM of Edwards (1995). 
Graphical models for time series

\section{The Continuous Case.}

Suppose one is dealing with random variables having continuous distributions.

\subsection{The Undirected Case.}

In the Gaussian case, conditional independence corresponds to zeroes in the inverse of the covariance matrix. Such models were studied in Dempster (1972) and are referred to as covariance selection. The model on the graph will have the conditional independences built in and estimates will need to reflect this. The inverse matrix corresponds to an undirected graph in the sense that the entry in row $u$, column $\nu$ being 0 corresponds to no edge $(u, \nu)$ in the graph whose nodes are all the labels. More specifically let $\left\{Y_{\nu}, \nu \varepsilon V\right\}$ be normal with covariance matrix $\Sigma$. Suppose vertices $u$ and $\nu$ are unconnected in the independence graph. The variates $Y_{u}$ and $Y_{\nu}$ are then statistically independent given the rest and

$$
\left(\Sigma^{-1}\right)_{u \nu}=0=R_{u \nu \mid r e s t}
$$

where $\mathbf{R}$ is the matrix of partial correlations. See Whittaker (1990) for a development of this result.

To examine the possibility of 0 partial correlation empirically, one can look for near 0 entries in an estimate $\widehat{R}$ of $\mathbf{R}$ or examine the change in deviance resulting from assuming first $R_{u \nu \mid r . e s t}$ is arbitrary and then that it is 0 .

\subsection{The Directed Case.}

On would work with joint densities factorizing as in (2.2) and examine the difference in deviance between the models with the edge present and absent, e.g. for Figure 4 the cases

$$
f\left(y_{1}, y_{2}, y_{3}\right)=f_{1}\left(y_{1}\right) f_{2}\left(y_{2} \mid y_{1}, y_{3}\right) f_{3}\left(y_{3} \mid y_{1}\right)
$$


or

$$
f\left(y_{1}, y_{2}, y_{3}\right)=f_{1}\left(y_{1}\right) f_{2}\left(y_{2} \mid y_{1}\right) f_{3}\left(y_{3} \mid y_{1}\right)
$$

\section{Discrete Case.}

\subsection{The Undirected Case.}

In the case that the random variables $Y_{\nu}, \nu \varepsilon V$, are discretevalued, undirected graphical models may be described by loglinear models. With $Y$ such that

$$
\operatorname{Prob}\{\mathrm{Y}=\mathrm{y}\}=\pi(\mathrm{y})
$$

one may write

$$
\log \pi(\mathbf{y})=\sum_{a} g_{a}\left(\mathbf{y}_{a}\right)
$$

where $a$ ranges over the subsets of $V$. Then $Y_{u}$ is independent of $Y_{\nu}$ given the rest is equivalent to $g_{a}=0$ if $\{u, \nu\}$ is in $a$, see Whittaker (1990), Edwards (1995).

The conditional independence hypothesis may again be examined by the edge exclusion deviance, that is the change in $-2 \log$ likelihood when the edge is removed from the graph.

\subsection{The Directed Case.}

Now one would work with conditional distributions, given parents, and a joint probability function as in (2.2) and in the case of Figure 4 for factorizations like (3.2.1).

\subsection{Extensions.}

There are mixed discrete/continuous models, see e.g. Lauritzen (1989), Whittaker (1990), Edwards (1995). In the contemporary 
research on this topic, given the discrete variables, the continuous variables are typically assumed jointly Gaussian.

\section{Time Series.}

Now random sequences and functions are under consideration.

\subsection{The Undirected Case.}

Consider the stationary vector-valued series $\mathrm{Y}(t)$ where $t$ is discrete or continuous. The strengh of association of the $u$-th and $\nu$-th components may be measure by the crosscorrelation function

$$
\operatorname{corr}\left\{Y_{u}(t+s), Y_{\nu}(t)\right\}
$$

as a function of lag $s$ or alternately by the coherency function

$$
R_{u \nu}(\lambda)=\lim _{T \rightarrow \infty} \operatorname{corr}\left\{\sum_{t} e^{-i \lambda t} Y_{u}(t), \sum_{t} e^{-i \lambda t} Y_{\nu}(t)\right\}
$$

as a function of frequency $\lambda$. As written, in (5.1.2), time is discrete and the sums are over $t=0, \cdots, T-1$. Both (5.1.1) and (5.1.2) are identically 0 in the case that the series $Y_{u}$ and $Y_{\nu}$ are statistically independent. The coherency matrix is a close analog of a correlation matrix being correlations of the components of frequency $\lambda$ in the series $Y_{u}$ and $Y_{\nu}$. see Brillinger (1975).

In the case of a stationary Gaussian time series $\{\mathrm{Y}(t)\}$, the mutual information of the components $Y_{u}$ and $Y_{\nu}$ is

$$
-\frac{1}{2 \pi} \int \log \left\{1-\left|R_{u \nu}(\lambda)\right|^{2}\right\} d \lambda
$$

see Pinsker (1964), Granger and Hatanaka (1964). The coherency function (5.1.2) occurs in this last expression. The measure is 0 when $R_{u \nu} \equiv 0$ and $\infty$ when $R_{u \nu} \equiv 1$. 
Suppressing the dependence on $\lambda$, the partial coherency of the series $Y_{u}$ and $Y_{\nu}$, removing the linear time invariant effects of the other series, is given by

$$
R_{u \nu \mid r e s t}=\left(R_{u \nu}-R_{u \mid r e s t} \bar{R}_{\nu \mid r e s t}\right) / \sqrt{\left(1-\left|R_{u \mid \text { rest }}\right|^{2}\right)\left(1-\left|R_{\nu \mid r e s t}\right|^{2}\right)}
$$

with the overbar denoting complex conjugate. This is the entry in row $u$, column $\nu$ of the inverse of the coherency matrix, $\mathbf{R}(\lambda)=\left[R_{u \nu}(\lambda)\right]$. It is a function of frequency, $\lambda$ and so distinguished from the continuous variate case of Section 3.1. This function will be identically 0 for stationary Gaussian $\mathbf{Y}$ when $Y_{u}$ and $Y_{\nu}$ are independent given the rest. References to the concept of conditional coherency include: Tick (1963), Granger and Hatanaka (1964), Brillinger and Hatanaka (1969, 1970), Gersch (1972).

Following Granger and Hatanaka (1964), the partial mutual information of $Y_{u}$ and $Y_{\nu}$ given the rest is

$$
-\frac{1}{2 \pi} \int \log \left\{1-\left|R_{u \nu \mid \text { rest }}(\lambda)\right|^{2}\right\} d \lambda
$$

and one sees the appearance of the partial coherency. Coherency is an acausal concept, being identically 1 if either series involved is a linear time invariant filtered version of the other, i.e. two-sided filters are allowed.

Sometimes one may wish to consider one-sided models and corresponding likelihoods. For example consider the stationary autoregressive Gaussian case. Suppose

$$
\mathbf{A}[\mathbf{Y}](t)=\mathbf{E}(t)
$$

with $\mathbf{Y}$ and $\mathbf{E} r$-vector-valued, with

$$
\mathbf{A}[\mathbf{Y}](t)=a(0) \mathbf{Y}(t)+a(1) \mathbf{Y}(t-1)+\cdots+a(p) \mathbf{Y}(t-p)
$$




\section{Graphical models for time series}

and with

$$
\operatorname{var} \mathbf{E}(t)=\sigma^{2} \mathbf{I}, \quad \operatorname{cov}\{\mathbf{E}(t+s), \mathbf{E}(t)\}=0 \quad \text { for } s \neq 0
$$

consider looking for 0 partial coherencies. The inverse of the spectral density matrix is

$$
2 \pi \overline{\mathbf{A}(\lambda)^{\tau}} \mathbf{A}(\lambda) / \sigma^{2}
$$

and one sees that the entry $u, \nu$ is 0 for all $\lambda$ if and only if

$$
\sum_{w=1}^{r} \sum_{0 \leq t+s, t \leq p} a_{w u}(t+s) a_{w \nu}(t)=0
$$

for all $s$.

The strength of connection in these two cases may be estimated by the change in deviance when model is fit both with some entries of $\mathbf{A}(\cdot)$ identically zero and not.

Some distributions and estimates appropriate to the problems considered in this section may be found in Andersen et al. (1995).

\subsection{The Directed Case.}

One would set down a joint density function appropriate to the situation. In the case of Figure 4 it might be derived from

$$
\begin{aligned}
& Y_{1} \text { free } \\
& Y_{2}(t)=\sum_{s \geq 1} a_{21}(s) Y_{1}(t-s)+\sum_{s \geq 1} a_{23}(s) Y_{3}(t-s)+\varepsilon_{2}(t) \\
& Y_{3}(t)=\sum_{s \geq 1} a_{31}(s) Y_{1}(t-s)+\varepsilon_{3}(t)
\end{aligned}
$$

with $\varepsilon_{2}(t)$ and $\varepsilon_{3}(t)$ independent of past values of $Y_{1}, Y_{2}, Y_{3}$. 


\subsection{The State Space Case.}

The book Harvey (1989) shows the ubiquity of the state space model in economics and econometrics. Vector ARMA's and ARMAX's are particular cases of this model. So too are models with complex, nonstationary trends and behavioral econometric models. Included too is the case of time varying parameters.

One formulation is provided by

$$
\begin{aligned}
\mathbf{S}_{t} & =\mathbf{A} \mathbf{S}_{t-1}+\mathbf{B X}_{t}+\mathbf{E}_{t} \\
\mathbf{Y}_{t} & =\mathbf{C S}_{t}+\varepsilon_{t}
\end{aligned}
$$

with $\mathbf{S}_{t}$ the unobserved state vector, $\mathbf{X}_{t}$ an exogenous series and $\mathbf{Y}_{t}$ the endogenous. The variates $\left(\mathbf{E}_{t}, \varepsilon_{t}\right)$ represent noise. The first equation is the transition equation, while the second is the observation.

This state space model may be usefully described by a graph, see Figure 5, and Normand and Tritcher (1992). The Kalman filter and algorithms developed by researchers in graphical modelling may be employed to predict and smooth $\mathrm{Y}_{t}$ here.

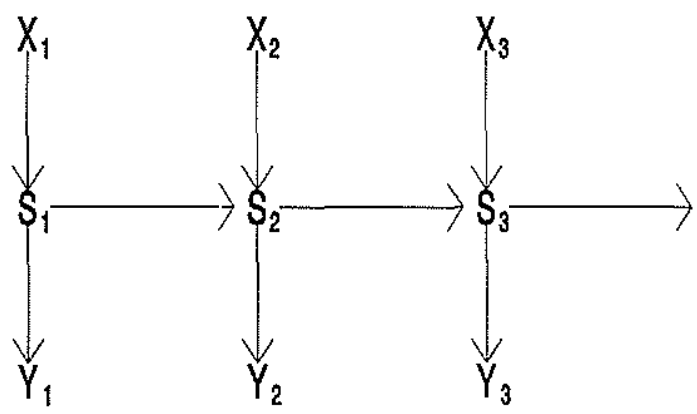

Figure 5. A directed graph representing the state model with the exogenous variable $\mathbf{X}_{t}$, the state variable $\mathbf{S}_{t}$ and the endogenous variable $\mathbf{Y}_{t}$. 


\section{Point Processes.}

The realizations of stochastic point processes are sets of points on the real line

$$
\cdots<\tau_{0}<\tau_{1}<\tau_{2}<\cdots .
$$

For example, the $\tau$ 's might correspond to time of: economic decisions, security sales or bankruptcies. A point process can also be described via counting functions, specifically with $I$ an interval one can set

$$
\begin{aligned}
N(I) & =\#\left\{\tau_{n} \text { in } I\right\} \quad N(t)=N(0, t] \\
d N(t) & =1 \text { if point in }(t, t+d t] \\
& =0 \text { otherwise. }
\end{aligned}
$$

References to point process analysis include: Snyder (1975), Daley and Vere-Jones (1988).

\subsection{Point Process Descriptions.}

Under general conditions, the conditional intensity function characterizes a vector-valued point process $\mathbf{N}$. It is given by the vector-valued variate $\left[\mu_{u}\left(t \mid H_{t}\right)\right]$ of

$$
\operatorname{Prob}\left\{d N_{u}(t)=1 \mid H_{t}\right\}=\mu_{u}\left(t \mid H_{t}\right) d t
$$

where $H_{t}=\{d \mathbf{N}(s), s \leq t\}$. The conditional intensity provides the likelihood and so is basic to the analysis of point process data, see Snyder (1975), Daley and Vere-Jones (1988).

\subsection{The Undirected Case.}

The crossproduct density, of the components $u$ and $\nu$ of the stationary point process $\mathbf{N}$, provides a measure of strength of association and is given by

$$
\operatorname{Prob}\left\{d N_{t}(t+u)=1 \text { and } d N_{\nu}(t)=1\right\} / d t d u \text {. }
$$


The association may also be studied via the coherency function

$$
R_{u \nu}(\lambda)=\lim _{T \rightarrow \infty} \operatorname{corr}\left\{\sum_{m} e^{-i \lambda \sigma_{m}}, \sum_{n} e^{-i \lambda \tau_{n}}\right\}
$$

where $\sum_{m}$ and $\sum_{n}$ sum over points of the two types available in $(0, T]$. These concepts are analogs of the crossvariance and coherency functions of time series mentioned above. There are also hybrid measures for time series and point processes.

The partial coherency of components $u$ and $\nu$, given the rest, is again defined by (5.1.3). One can make two arguments for considering the partial coherency as a measure of conditional independence in the point process case. First, the empirical Fourier transforms appearing in (6.1.2) satisfy a Central Limit Theorem, i.e. are approximately normal and one can look to the inverse of their covariance matrix as a measure of conditional dependence. As an alternate argument, suppose that it is possible to write

$$
d N_{u}(t)=\left[\sum_{j \neq u, \nu} \int a_{j}(t-s) d N_{j}(s)\right] d t+d \Gamma_{u}(t)
$$

with $\Gamma_{u}$ a noise process such that

$$
E\left\{d \Gamma_{u}(t) \mid N_{j}, j \neq u, \nu\right\}=0
$$

and

$$
\operatorname{cov}\left\{d \Gamma_{u}(s), d \Gamma_{\nu}(t)\right\}=0
$$

then the partial coherency of $Y_{u}$ and $Y_{\nu}$ will be 0 .

\subsection{Directed Case.}

Suppose there is a directed network with three nodes having point processes as in Figure 4. The arrows now mean that, for example, the behavior of process 2 at time $t$ depends on the past of the 
other two processes. A structural model leading to associated point processes $Y_{1}$ and $Y_{2}$ in the presence of a third process $Y_{3}$, follows. Write $Y_{1}=\left\{\sigma_{m}\right\}$ and $Y_{3}=\left\{\gamma_{0}\right\}$ and

$$
U(t)=\sum_{\sigma_{m}<t} a_{21}\left(t-\sigma_{m}\right)+\sum_{\gamma_{0}<t} a_{23}\left(t-\gamma_{0}\right)
$$

for summation functions $a_{21}(\cdot)$ and $a_{23}(\cdot)$. Suppose now that a $Y_{2}$ point occurs at time $t$ when $U(t)$ upcrosses a random threshold $\theta(t)$. The question of whether $Y_{2}$ and $Y_{1}$ are independent given $Y_{3}$ in this case may be phrased as: is $a_{23}(\cdot) \equiv 0$ ? Given data this question may be addressed by fitting the model both with and without $a_{23}(\cdot)$ present.

As an approximation to the likelihood based on the conditional intensity one procedure is to replace the point processes here by 0 1 discrete time series $M_{t}, N_{t}, O_{t}$, and to estimate parameters by maximizing the log likelihood

$$
\sum_{t}\left[N_{t} \log \Phi\left(U_{t}-\mu\right)+\left(1-N_{t}\right) \log \left(1-\Phi\left(U_{t}-\mu\right)\right)\right]
$$

where, for example, $\Phi(\cdot)$ is the standard normal cumulative, and

$$
U_{t}=\sum_{u=1}^{\infty} m_{t-u} M_{u}+\sum_{u=1}^{\infty} o_{t-u} O_{u}
$$

and

$$
\theta_{t}=\mu+\varepsilon_{t} .
$$

Here it may be assumed that noise values $\varepsilon_{t}$ are independent identically distributed normals. The strength of the edge $3 \rightarrow 2$ may be measured by the change in deviance occurring when the function $o_{t}$ is set to 0 identically. 
For related models and empirical analyses, motivated by biological neuron networks see Brillinger (1991, 1995).

Explanatory variables may be included, e.g. one corresponding to the presence of an intervention. Set $S(t)=1$ when the intervention is present and $=0$ otherwise. One might assume

$$
d N_{u}(t)=1 \text { if } \alpha S(t)+\sum_{\nu \neq u} \int_{0}^{\infty} a_{u \nu}(t-s) d N_{\nu}(s) \text { upcrosses } \theta_{u}(t)
$$

As a measure of the strength of connection one can consider the change in deviance resulting from refitting with some $a_{u \nu}(\cdot)=0$.

\section{Difficulties.}

There may be unmeasured variates or measurement error, see Robinson (1986). Interpretations can be fraunt with difficulties, as in the case of path analysis, see Freedman (1987). Feedback may be present, complicating the construction of the likelihood function.

\section{Discussion and Summary.}

In distinction with the ordinary random variable case, the time series and point process cases lead to the consideration of parameters that are functions, functions of frequency or lag.

Graphical representations have been found to be valuable in the sense that they force one to think hard about the characters of the dependencies involved. Such considerations are basic to economics and econometrics, see Hendry and Richards (1983). Econometricians are often lead to think about the issue of causality, see e.g. Geweke (1984), and the current work on causality and graphs, see e.g. Pearl (1995) and its discussion, has pertinent specific techniques to offer.

The referee made the basic point that temporal systems can be time-varying, the graph may change (as an example I mention that 
nodes may appear or disappear) and a more sophisticated structure is needed. I agree and thank the Referee for this and other comments.

Experimental systems can provide a testbed for econometric techniques. The techniques presented here have been validated, to an extent, by experimental data from neurophysiology, see Rosenberg et al. (1989), Brillinger (1991, 1995).

Submetido em Outubro de 1995. Revisado em Fevereiro de 1996.

\section{Referências}

Andersen, H.; Hojbjerre, H.; D. Sorensen \& P.S. Eriksen. 1995. "Linear and Graphical Models". New York: Springer.

Brémaud, P. 1981. "Point Processes and Queues". New York: Springer.

Brillinger, D.R. 1975. "Time Series: Data Analysis and Theory". New York: Holt Rinehart.

Brillinger, D.R. 1991. "Nerve cell spike train data analysis: a progression of tchnique." J. Amer. Statist. 87: 260-271.

Brillinger, D.R. 1995. "Networks of biological time series and puini processes." In Morgenthaller S., John W. Tukey Festschrift. To appear.

Brillinger, D.R. \& M. Hatanaka. 1969. "An harmonic analysis of nonstationary multivariate economic processes." Econometrica. 131-141.

Brillinger, D.R. \& M. Hatanaka. 1970. "A permanent income hypothesis relating to the aggregate demand for money (an application of spectral and moving spectral analysis)." Economic Studies Quarterly. 21: 44-71.

Cox, D.R. \& N. Wermuth. 1993. "Linear dependencies represented by chain graphs." Statistical Science. 8: 204-218. 
Daley, D. J. \& D. Vere- Jones. 1988. "An Introduction to the Theory of Point Processes". New York: Springer.

Dempster, A.P. 1972. "Covariance selection." Biometrics. 28: 157175.

Edwards, D. 1995. "Introduction on Graphical Modelling". New York: Springer.

Freedman, D.A. 1987. "As others see us: a case study in path analysis." J. Educational Stat. 12: 101-128.

Geiger, D. \& J. Pearl. 1993. "Logical and algorithmic properties of conditional independence and graphical models." Ann. Statist. 21: 2001-2021.

Gersch, W. 1972. "Causality or driving in electrophysiological signal analysis." Math. Biosci. 14: 177-196.

Geweke, J. 1984. "Inference and causality in economic time series models." Z. Griliches and M.D. Intriligator, Handbook of Econometrics, vol. II. Amsterdam: Elsevier.

Glymour, C.; R. Scheines, P. Spirtes \& K. Kelly. 1987. "Discovering Causal Structure". New York: Academic.

Granger, C.W.J. \& M. Hatanaka. 1964. "Spectral Analysis of Economic Time Series". Princeton: Princeton Press.

Granger, C.W. J. 1980. "On the synthesis of time series and econometric models." In Brillinger, D.R. and Tiao, G.C., Directions in Time Series. Hayward, Institute of Mathematical Statistics.

Harvey, A.C. 1989. "Forecasting, Structural Time Series Models and the Kalman Filter". Cambridge: Cambridge University Press.

Hendry, D.F. \& Richard, J.F. 1983. "The econometric analysis of economic time series." Int. Statist. Review. 51: 111-148.

Jacquez, J.A. 1972. "Compartmental Analysis in Biology and Medicine". Amsterdam: Elsevier. 
Graphical models for time series

Kanter, M. 1979. "Lower bounds for nonlinear prediction error in moving average processes." Ann. Prob. 7: 128-138.

Kiiveri, H. \& T.P. Speed. 1982. "Structural analysis of multivariate data: a review." In Leinhardt, S. Sociological Methodology. San Francisco: Jossey-Bass.

Lauritzen, S. J. 1989. "Mixed graphical association models." Scand. J. Statist. 16: 273-306.

Lauritzen, S.L.; A.P. Dawid; B.N. Larsen \& H.G. Leimer. 1990. "Independence properties of directed Markov fields." Networks. 20: 491-505.

Normand, S.L. \& D. Trichler. "Parameter updating in a Bayes network." J. American Statistical Assoc. 87: 1109-1115.

Parzen, E. 1983. "Time series model identification by estimating information." In Karlin, S.; Amemiya, T. and Goodman, L.A. Studies in Econometrics, Time Series and Multivariate Statistics. New York: Academic.

Pearl, J. "Causal diagrams for empirical research." Biometrika. 82: 669-688.

Philips, A.W. 1950. "Mechanical models in economic dynamics." Economica. 17: 282-305.

Pinsker, M.S. 1964. "Information and Information Stability of Random Variables and Processes". San Francisco: Holden-Day.

Robinson, P.M. 1986. "On the errors-in-variables problem for time series". J. Mult. Analysis. 19: 240-250.

Rosenberg, J.R.; A.M. Amjad; P. Breeze, D.R. Brillinger \& D.M. Haliday. 1989. "The Fourier approach to the identification of functional coupling between neuronal spike trains." Prog. Biophys. Molec. Biol. 53: 1-31.

Smith, J.Q. 1989. "Influence diagrams for statistical modelling." Ann. Statist. 17: 654-672.

Synder, D.L. 1975. "Random Point Processes". New York: Wiley. 
Swade, D. 1995. "The Philips economic computer." Resurection. 12: 11-18.

Tick, L.J. 1963. "Conditional spectra, linear systems and coherency." In Rosenblatt, M. Time Series Analysis. New York: Wiley.

Tinbergen, J. 1939. "Business Cycles in the United States of America. 1919-1932". Geneva: League of Nations.

Wahba, G. 1971. "Some tests of independence of stationary multivariate time series." J. Royal Statist. Soc. B. 33: 153-166.

Wermuth, N. 1992. "On block-recursive linear regression equations." REBRAPE. 6: 1-56.

Wermuth, N. \& Lauritzen, S.L. 1990. "On substantive research hypotheses, conditional independence gr phs and graphical chain models." J. Royal Statist. Soc. B. 52: 21-72.

Whittaker, J. 1990. "Graphical Models in Applied Mathematical Multivariate Statistics". New York: Wiley.

Wold, H. 1956. "Causal inference from observational data." J. Roy. Statist. Soc. A. 119: 28-61. 
